\title{
Clopidgrel Resistance and Periprocedual Thromboembolic Complications in Neuroendovascular Treatment
}

Satoshi Shitara and Yoshinori Akiyama

Objective: Antiplatelet therapy is essential in neuroendovascular therapy to avoid periprocedural thromboembolic events. The purpose of our study was to evaluate the relationship between platelet aggregation analysis by VerifyNow assay system (Accumetrics, San Diego, CA, USA) and thromboembolic complications.

Methods: Seventy-two neuroendovascular procedures were performed between March 2013 and February 2015 in this institution. There were 41 males (56.9\%) and their mean age was 69.9 years. Meanwhile, there were 31 females $(43.0 \%)$ and their mean age was 62.7 years. In all cases, clopidogrel (CLP) was used as one of the periprocedural antiplatelet therapy. All patients were measured CLP response as P2Y12 Reaction Units (PRU) value using VerifyNow assay system (Accumetrics, San Diego, CA, USA) with the whole blood sample collected from the long sheath located at the femoral artery. All patients received diffusion-weighted image (DWI) on MRI at 1-4 days after the procedure. The occurrence of thromboembolic events was recorded and the relationship between CLP response and DWI hyper-intensity area (HIA) positive was analyzed. Appropriate cut-off value to predict thromboembolic events was examined.

Results: Symptomatic thromboembolic complications occured in three patients (4.1\%), and each PRU value was all over 226. CLP hypo-responder in our study recognized 46 patients $(63.8 \%)$ as cut-off PRU value set 208 . As the result of cut-off value defined as CLP hypo-responder, with regard to PRU more than 208, a significant difference appear in DWI HIA positive or negative, but with regard to PRU more than 230, a significant difference is not recognized.

Conclusion: In our study, CLP response measured by VerifyNow assay system is well correlated with the periprocedural thromboembolic events in neuroendovascular procedure. The cut-off value of PRU in neuroendovascular therapy is not still defined, but CLP hypo-responder defined PRU value more than 208 might be meaningful in neuroendovascular therapy performed to Japanese. We should make the custom-made periprocedural anti-platelet therapy according to personal response.

Keywords \ antiplatelet therapy, neuroendovascular therapy, VerifyNow assay system

\section{Introduction}

Recently, the appearance of various techniques and devices in neuroendovascular treatment has increased the risk of vascular intima injury. Therefore, antiplatelet therapy is essential for preventing thromboembolic complications in

Department of Neurosurgery, Tenri Hospital

Received: November 4, 2015; Accepted: December 6, 2015

Corresponding author: Satoshi Shitara. Department of Neurosurgery, Tenri Hospital, 200 Mishima-cho, Tenri City, Nara 632-8552, Japan

Email: s-shitara@umin.ac.jp

(C)2016 The Editorial Committee of Journal of Neuroendovascular Therapy. All rights reserved. neuroendovascular treatment. A simple monitoring system, the VerifyNow system (Accumetrics, San Diego, CA, USA), has been introduced. ${ }^{1-12)}$ According to recent studies, Asians sometimes do not respond to clopidogrel (CLP). ${ }^{13,14)}$ In this study, we examined the association between the results of measurement and thromboembolic complications and its clinical significance in patients who underwent neuroendovascular treatment after the introduction of the VerifyNow system in our hospital.

\section{Subjects and Methods}

Of the 105 patients who underwent neuroendovascular treatment in our hospital in March 2013, when the VerifyNow 
system was introduced in our hospital, we retrospectively investigated the association between $\mathrm{P} 2 \mathrm{Y} 12$ reaction units (PRU) and perioperative ischemic complications within 30 days after surgery, with the appearance of a hyper-intensity area (HIA) on diffusion-weighted imaging (DWI) in 72 patients in whom CLP was used for perioperative antiplatelet therapy. For imaging, a MAGNETOM Skyra 3 tesla system (SIEMENS, segmented-EPI-DWI, TR/TE510/68 and 112, FOV: $220 \mathrm{~mm}$, matrix: $160 \times 136,5.0 \mathrm{~mm} / 1.0 \mathrm{~mm}$, scan time: $93 \mathrm{sec}$ ) was used. Patients with one or more HIAs were regarded as showing positive findings.

The 72 patients consisted of 42 who underwent carotid artery stenting (CAS) and 30 who underwent unruptured cerebral aneurysm coiling. The patient background is shown in Table 1. In all patients, the femoral artery was punctured under local anesthesia. An $8 \mathrm{Fr}$ sheath was inserted for CAS, and a $7 \mathrm{Fr}$ sheath for coiling. Before the intravenous injection of heparin at 5000 units, whole blood was collected through the sheath. After 30 minutes, PRU measurement was performed using the VerifyNow system. For CAS, a FilterWire EZ device (Boston Scientific, Natick, MA, USA) was used as a distal embolization-preventing device in all patients. PRECISE stents (Cordis, Johnson \& Johnson, Fremont, CA, USA) were selected for 35 patients, a Carotid WALLSTENT (Boston Scientific, Natick, MA, USA) for 1, and PROTÉGÉ stents (eV3 Covidien, Irvine, CA, USA) for 6 . For patients in whom unstable plaques were strongly suspected based on various preoperative modalities, proximal protection was adopted, and they were excluded from the subjects of this study. To unify the conditions, an Rx-Genity balloon (Kaneka Medics, Osaka) for pre-dilation and an AVIATOR Plus balloon (Cordis, Johnson \& Johnson, Fremont, CA, USA) for post-dilation were used for percutaneous transluminal angioplasty. In all patients, dilation was performed before and after stenting.

In all patients, coiling was performed using a balloonassisted technique with a HyperForm (eV3 Covidien, Irvine, CA, USA).

In the 72 subjects, the oral administration of antiplatelet agents was started at least 5 days before surgery. During surgery, systemic heparinization was conducted, with a target activated clotting time (ACT) of 300. After surgery, argatroban was administered for 48 hours $(60 \mathrm{mg} /$ day). Subsequently, the divided administration of argatroban $(20 \mathrm{mg} /$ day, three hours each in the morning and evening) was continued for 5 days.

To evaluate CLP hypo-responders, a PRU level of more than 208, which was adopted according to the ACCF/AHA
2011 guidelines, and that of more than 230, which was examined in the GRAVITAS trial, was used as cut-off values. In the subjects, we investigated the correlation between the appearance of perioperative symptomatic complications and PRU level, as well as that between the proportion of patients with one or more HIAs on postoperative MRI (DWI) and PRU level, using Fisher's test. ${ }^{15,16)}$

\section{Results}

Hemorrhagic complications were observed in two patients: hemothorax and hematoma at the puncture site. The two patients were CLP hyper-responders, with a PRU level of under 95. However, they were discharged based on a modified Ranking Scale (mRS) score of 0. Embolic complications were noted in three patients (4.1\%), coiling: two patients (Cases 1 and 2), CAS: 1 (Case 3). These patients were CLP hypo-responders, with a PRU level of more than 208. In Case 1 (PRU: 296), an unruptured aneurysm was detected in the $\mathrm{C} 3$ region of the right internal carotid artery. At the completion of coiling, angiography revealed a shadow defect in an area adjacent to the aneurysmal neck. The additional intravenous injection of heparin at 2000 units was performed, and 5 $\mathrm{mg}$ of Ozagrel Sodium was slowly injected into the artery, leading to the disappearance of the thrombus. From the day of surgery, cilostazol (CSZ) at $200 \mathrm{mg} /$ day was additionally administered. The patient was discharged, with a mRS score of zero (Fig. 1). In Case 2 (PRU: 226), an aneurysm was detected in the $\mathrm{C} 3$ region of the left internal carotid artery. Motor dysfunction of the right fingers appeared a few hours after the completion of surgery. MRI (DWI) revealed an HIA in the left cerebral hemisphere motor area. Finally, rehabilitation was required, and the patient was referred to another hospital for rehabilitation, with a mRS score of 2 (Fig. 2). In Case 3 (PRU: 259), a Carotid WALLSTENT was inserted to treat radiation-induced stenosis of the left internal carotid artery after radiotherapy for oral floor cancer. After surgery, motor dysfunction of the right fingers was gradually observed, but symptoms were mild. CSZ at $200 \mathrm{mg}$ /day was additionally administered. The patient was discharged, with a mRS score of 2 (Fig. 3).

The proportion of patients with one or more HIAs on DWI in the 72 subjects (cut-off value of PRU: 208 or 230) is shown in Table 2. When regarding a PRU level of more than 208 was regarded as a cut-off value, there were 47 non-responders to CLP $(63.8 \%)$. When a PRU level of more than 230 was regarded as a cut-off value, there were 37 non-responders to CLP (51.3\%). In contrast, when an aspirin 


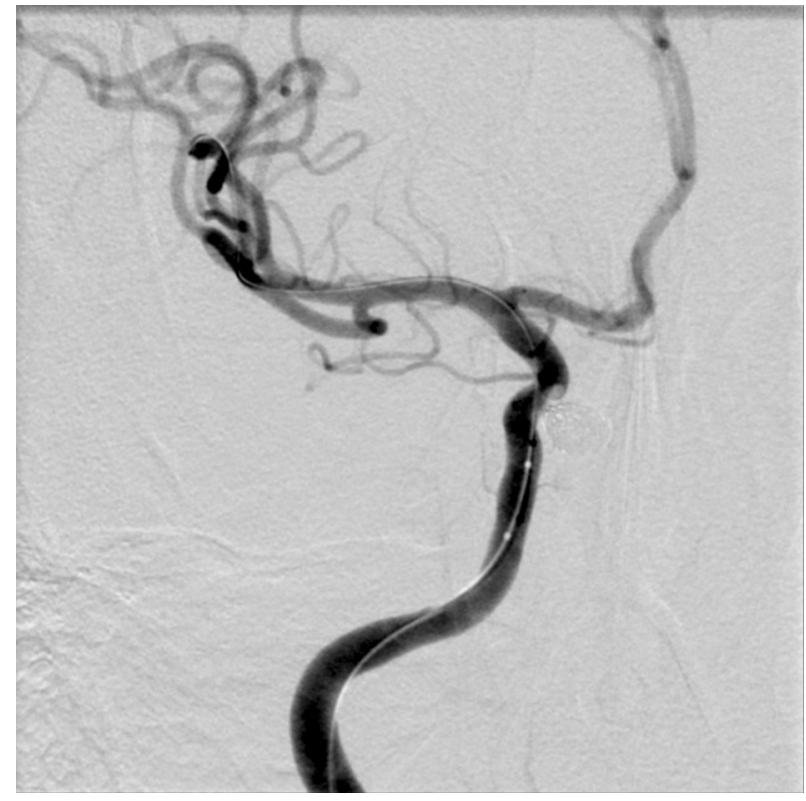

Fig. 1 A case of intraprocedural thrombosis during coiling to right internal carotid artery unruptured aneurysm. Thrombus formation was seen at the distal portion of the aneurysmal neck.

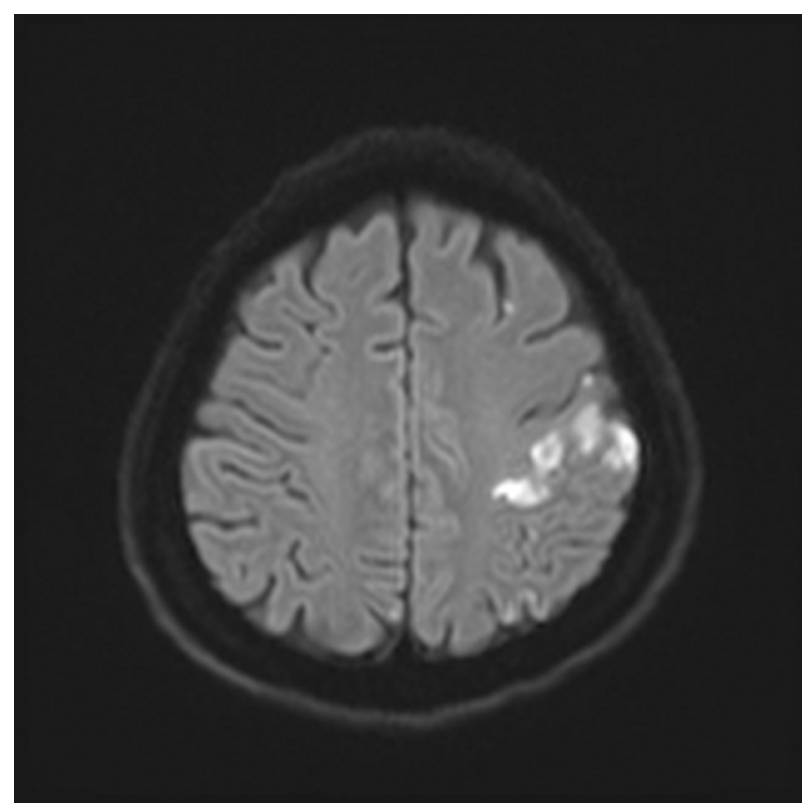

Fig. 2 A case of thromboembolic events seen in coiling procedure. Hyper-intensity signal were seen at the motor hand cortex after few hours when coiling finished.

reaction unit (ARU) level of 550 was regarded as a cut-off value, only one (1.4\%) of 70 patients was a non-responder to aspirin. When defining patients with a PRU level of more than 208 as CLP hypo-responders, there was a correlation between the proportion of patients with one or more HIAs on DWI and PRU level $(\mathrm{P}=0.0001$, Fisher's probability

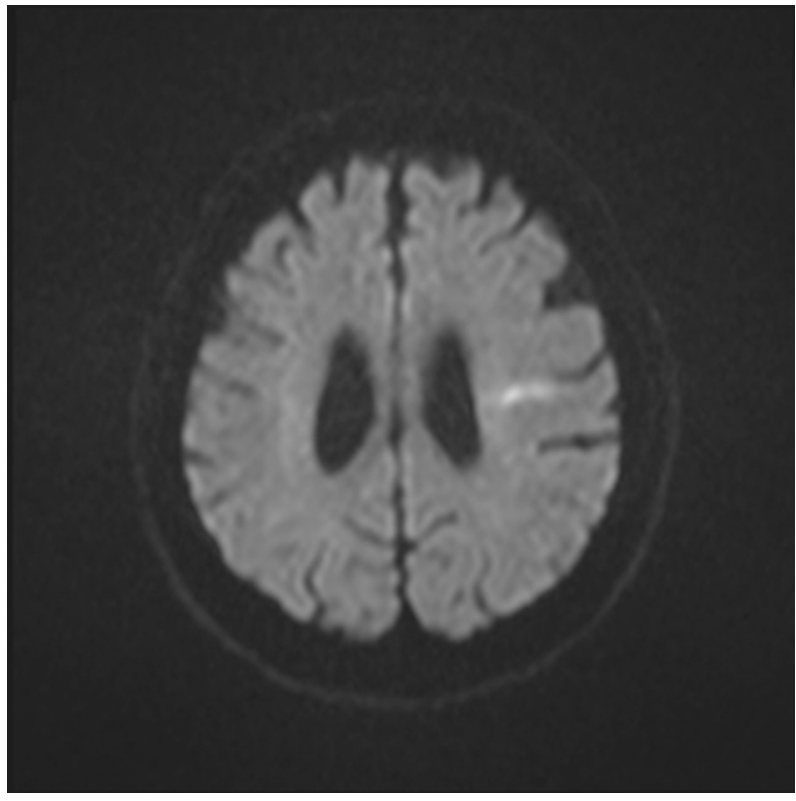

Fig. 3 A case of thromboembolic events seen in carotid artery stenting procedure. Hyper-intensity signal were seen at the motor hand cortex, and disorder of coordinated movement gradually appeared.

test). When defining those with a PRU level of more than 230 as CLP hypo-responders, the p-value was 0.2457 . There was a correlation between the proportion of patients with one or more HIAs on DWI and the CLP response rate only when defining those with a PRU level of more than 208 as CLP hypo-responders (Table 2). When defining patients with a PRU level of more than 208 as CLP hypo-responders, the p-values in the CAS and coiling groups were 0.0101 and 0.0109 , respectively. When defining those with a PRU level of more than 230 as CLP hypo-responders, the p-values were 0.7579 and 0.2602 , respectively. There was a correlation when defining patients with a PRU level of more than 208 as CLP hypo-responders, as above (Table 2).

\section{Discussion}

CLP is routinely used in the field of cerebrovascular treatment in Japan. However, there are individual differences in its inhibitory effects on platelet aggregation. ${ }^{7)}$ As the mechanism, it has been shown that the functional loss of an allele of the CYP2C19 gene, as a drug-metabolizing enzyme, contributes to the outcome. Furthermore, this allele is particularly frequent in Asians. ${ }^{13,14,17)}$

Factors involved in CLP resistance include poor compliance, excessively low-dose administration, drug interactions and individual differences in intestinal absorption/liver 
Table 1 Baseline demographic and clinical characteristics

\begin{tabular}{ccc} 
& CAS N $=42$ & Aneurysm N=30 \\
Age (y.o.) & $71.3 \pm 6.92$ & $60.7 \pm 11.0$ \\
Sex female - no. (\%) & $6(14.2)$ & $25(83.3)$ \\
Hypertension - no. (\%) & $32(76.1)$ & $7(23.3)$ \\
Diabetes mellitus - no. (\%) & $14(33.3)$ & $2(6.6)$ \\
Hyperlipidemia - no. (\%) & $25(59.5)$ & $5(16.6)$ \\
<Antiplatelets & & \\
TAPT - no. (\%) & $3(7.1)$ & $0(0)$ \\
DAPT $\quad$ CLP + ASA - no. (\%) & $37(88.0)$ & $30(100)$ \\
\hline
\end{tabular}

CAS: carotid artery stenting; TAPT: triple antiplatelet therapy; DAPT: dual antiplatelet therapy; CLP: clopidogrel 75 mg/day; ASA: aspirin 100 mg/day; CSZ: cilostazol $200 \mathrm{mg} /$ day

Table 2 Incidence of hyper-intensity areas on DWI

\begin{tabular}{lccc} 
Total & DWI positive & & DWI positive \\
\hline $\mathrm{PRU}<208$ & $4 / 26(15.3 \%)$ & $\mathrm{PRU}<230$ & $13 / 35(37.1 \%)$ \\
$\mathrm{PRU}>208$ & $28 / 46(60.8 \%)$ & $\mathrm{PRU}>230$ & $19 / 37(51.3 \%)$ \\
$\mathrm{p}=0.0001^{*}$ & $32 / 72$ & $\mathrm{P}=0.2457$ & $32 / 72$ \\
\hline $\mathrm{CAS}$ series & DWI positive & & DWI positive \\
$\mathrm{PRU}<208$ & $4 / 16(25.0 \%)$ & $\mathrm{PRU}<230$ & $10 / 21(47.6 \%)$ \\
$\mathrm{PRU}>208$ & $18 / 26(69.2 \%)$ & $\mathrm{PRU}>230$ & $12 / 21(57.1 \%)$ \\
$\mathrm{P}=0.0101^{*}$ & $22 / 42$ & $\mathrm{P}=0.7579$ & $22 / 42$ \\
$\mathrm{Coiling} \mathrm{series}$ & $\mathrm{DWI}$ positive & & DWI positive \\
$\mathrm{PRU}<208$ & $0 / 10(0.0 \%)$ & $\mathrm{PRU}<230$ & $3 / 14(21.4 \%)$ \\
$\mathrm{PRU}>208$ & $10 / 20(50.0 \%)$ & $\mathrm{PRU}>230$ & $7 / 16(43.7 \%)$ \\
$\mathrm{P}=0.0109^{*}$ & $10 / 30$ & $\mathrm{P}=0.2602$ & $10 / 30$ \\
\hline
\end{tabular}

"statistically signify $(\mathrm{P}<0.05)$. DWl: diffusion weighted image; PRU: P2Y12 reaction units; CAS: carotid artery stenting

metabolism, platelet hyperactivity related to diabetes/acute coronary disorder/acute infarction, and CYP2C19 gene variation. Therefore, CLP hypo-responders must be considered from multiple viewpoints. However, in this study, the number of patients was small. In future, a large-scale clinica study should be conducted in Japan.

Of the 72 patients, $46(63.8 \%)$ were regarded as CLP hypo-responders when establishing a PRU level of more than 208 as a cut-off value. In previous studies, the percentage ranged from 5 to $65 \%$; our data may be useful. Lee et al. reported the correlation between CLP hypo-responders and embolic complications. As the proportion of CLP hyporesponders in Asians is high, there would be high risks in using CLP as perioperative antiplatelet therapy for neuroendovascular treatment in Japan. On the other hand, Prasugrel, which was approved in Europe and the United States in 2009 , is an ADP receptor inhibitor that belongs to the thienopyridine system, as reported for CLP, but its ADP-induced inhibitory effects on platelet aggregation are faster and more accurate than those of CLP. The effects appear even in the absence of CYP2C19. In Japan, Prasugrel became clinically available in the field of cardiology in May 2014. In future, its application might be approved in the cerebrovascular field. Of the 70 patients, $1(1.4 \%)$ did not respond to aspirin based on the ARU level, which was simultaneously measured. This agent may universally exhibit antiplatelet actions. It would be appropriate for perioperative antiplatelet therapy in Japan.

Light transmittance aggregometry (LTA) is a standard for other methods to measure platelet aggregation. Breet et al. suggested that the VerifyNow system is more useful than LTA for predicting the mortality rate and incidences of myocardial infarction/stent thrombosis/ischemic stroke within 1 year. This system facilitates simple measurement. In future, it should be routinely used as a platelet aggregation-measuring device in clinical practice in Japan. ${ }^{9)}$

In the field of cardiology, it has been indicated that the risk of complications is significantly higher in patients with a PRU level of under 95 and those with that of more than 208 (ACCF/AHA 2011 guidelines). However, an accurate cut-off value has not yet been established in Japan. ${ }^{15)}$ In this study, we compared two cut-off values: a PRU level of more than 208, which was adopted according to the ACCF/AHA 2011 guidelines, and that of more than 230 , which was examined 
in the GRAVITAS trial conducted by Accumetrics. ${ }^{15,16)}$ It was reported that HIAs on DWI after neuroendovascular treatments were observed in more than $30 \%$ of patients. However, in this study involving procedure standardization, there was a significant difference in the proportion of patients with one or more HIAs on postoperative DWI when establishing a PRU level of more than 208 as a cut-off value. ${ }^{18)}$ Postoperative embolic and hemorrhagic complications were noted in CLP hypo- and hyper-responders (cut-off value of PRU: under 95 and more than 208, respectively). Values measured using the VerifyNow system may be useful for predicting postoperative complications.

In this study, there was a correlation between the PRU level and proportion of patients with one or more HIAs on postoperative DWI, suggesting the usefulness of the perioperative measurement of platelet aggregation using the VerifyNow system for reducing the incidence of embolic complications. This study has some limitations: the number of patients was limited; and the association with various factors, such as medical history, proton pump inhibitors, aneurysm size/site, and carotid plaque features, was not analyzed. However, in future, measurement should be performed prior to the day before treatment without predicting the postoperative outcome based on data on the day of surgery. When perioperative antiplatelet therapy is not considered effective, treatment may be postponed to switch drugs; tailor-made perioperative antiplatelet therapy may be performed based on measurement using the VerifyNow system.

\section{Conclusion}

When establishing a PRU level of more than 208 as a cutoff value for CLP hypo-responders using the VerifyNow system, there was a significant difference in the proportion of patients with one or more HIAs on DWI between the CAS and coiling groups, suggesting that measurement using the VerifyNow system is useful for predicting postoperative DWI findings and the development of embolic complications.

It is not possible to measure individuals' responses to CLP using the VerifyNow system in all hospitals of Japan, but, in future, a large-scale clinical study should be conducted to establish an accurate cut-off value in Japan.

\section{Disclosure Statement}

There is no conflict of interest to be disclosed.

\section{References}

1) Delgado Almandoz JE, Crandall BM, Scholz JM, et al. Last-recorded P2Y12 reaction units value is strongly associated with thromboembolic and hemorrhagic complications occurring up to 6 months after treatment in patients with cerebral aneurysms treated with the pipeline embolization device. AJNR Am J Neuroradiol 2014; 35: 128-35.

2) Fifi JT, Brockington C, Narang J, et al. Clopidogrel resistance is associated with thromboembolic complications in patients undergoing neurovascular stenting. AJNR Am J Neuroradiol 2013; 34: 716-20.

3) Pandya DJ, Fitzsimmons BF, Wolfe TJ, et al. Measurement of antiplatelet inhibition during neurointerventional procedures: the effect of antithrombotic duration and loading dose. J Neuroimaging 2010; 20: 64-9.

4) Prabhakaran S, Wells KR, Lee VH, et al. Prevalence and risk factors for aspirin and clopidogrel resistance in cerebrovascular stenting. AJNR Am J Neuroradiol 2008; 29: 281-5.

5) Lee DH, Arat A, Morsi H, et al. Dual antiplatelet therapy monitoring for neurointerventional procedures using a point-ofcare platelet function test: a single-center experience. AJNR Am J Neuroradiol 2008; 29: 1389-94.

6) Goh C, Churilov L, Mitchell P, et al. Clopidogrel hyperresponse and bleeding risk in neurointerventional procedures. AJNR Am J Neuroradiol 2013; 34: 721-6.

7) Haraguchi K, Miyachi S, Izumi T, et al. Resistance to antiplatelet agents assessed by a point-of-care platelet function test and thromboembolic adverse events in neurointervention. No Shinkei Geka 2012; 40: 399-406.

8) Kang HS, Kwon BJ, Kim JE, et al. Preinterventional clopidogrel response variability for coil embolization of intracranial aneurysms: clinical implications. AJNR Am J Neuroradiol 2010; 31: 1206-10

9) Breet NJ, van Werkum JW, Bouman HJ, et al. High on-treatment platelet reactivity to both aspirin and clopidogrel is associated with the highest risk of adverse events following percutaneous coronary intervention. Heart 2011; 97: 983-90.

10) Paniccia R, Antonucci E, Maggini $N$, et al. Comparison of methods for monitoring residual platelet reactivity after clopidogrel by point-of-care tests on whole blood in highrisk patients. Thromb Haemost 2010; 104: 287-92.

11) Feher G, Feher A, Pusch G, et al. Clinical importance of aspirin and clopidogrel resistance. World J Cardiol 2010; 2: 171-86.

12) Lee DH, Arat A, Morsi H, et al. Dual antiplatelet therapy monitoring for neurointerventional procedures using a point-ofcare platelet function test: a single-center experience. AJNR Am J Neuroradiol 2008; 29: 1389-94.

13) Goldstein JA, Ishizaki T, Chiba K, et al. Frequencies of the defective CYP2C19 alleles responsible for the mephenytoin 
poor metabolizer phenotype in various Oriental, Caucasian, Saudi Arabian and American black populations. Pharmacogenetics 1997; 7: 59-64.

14) Kubota $T$, Chiba $K$, Ishizaki T. Genotyping of S-mephenytoin 4'-hydroxylation in an extended Japanese population. Clin Pharmacol Ther 1996; 60: 661-6.

15) Wright RS, Anderson JL, Adams CD, et al. 2011 ACCF/ AHA focused update of the Guidelines for the Management of Patients with Unstable Angina/Non-ST-Elevation Myocardial Infarction (updating the 2007 guideline): a report of the American College of Cardiology Foundation/American Heart Association Task Force on Practice Guidelines developed in collaboration with the American College of Emergency Physicians, Society for Cardiovascular Angiography and
Interventions, and Society of Thoracic Surgeons. J Am Coll Cardiol 2011; 57: 1920-59.

16) Price MJ, Berger PB, Teirstein PS, et al.: GRAVITAS Investigators. Standard- vs high-dose clopidogrel based on platelet function testing after percutaneous coronary intervention: the GRAVITAS randomized trial. JAMA 2011; 305: 1097-105.

17) Alván G, Bechtel P, Iselius L, et al. Hydroxylation polymorphisms of debrisoquine and mephenytoin in European populations. Eur J Clin Pharmacol 1990; 39: 533-7.

18) Cronqvist M, Wirestam R, Ramgren B, et al. Diffusion and perfusion MRI in patients with ruptured and unruptured intracranial aneurysms treated by endovascular coiling: complications, procedural results, MR findings and clinical outcome. Neuroradiology 2005; 47: 855-73. 